\title{
Derechos humanos en las estructuras monetarias y políticas actuales
}

Conferencia con motivo del VII Seminario Internacional del programa de diálogo filosófico Norte-Sur sobre el tema "Filosofia, teologia, política y economía en contexto de la lucha entre pluralismo cultural y universalización de los derechos humanos", en la Universidad Centroamericana “José Simeón Cañas" (UCA) de San Salvador Del 26 al 30 de julio de 1998.

\section{Introducción}

En el contexto de los procesos de individualización en nuestra sociedad, de la consiguiente separación del ser humano de los ámbitos colectivos tradicionales, y en el trasfondo de la experiencia multicultural de la internacionalización y globalización transmitidas por los medios de comunicación, surge entre los seres humanos una gran necesidad de orientación. El deseo ampliamente difundido, de sentido y de orden, se ve reforzado por el hecho de que la modernidad no ha cumplido sus promesas de proporcionar una interpretación y una organización racional de la vida, libre de toda protección metafísica. La existencia moderna parece estar marcada por la conciencia de que en nuestras ordenaciones sociales, religiosas y culturales carecen de una obviedad', de un carácter definitivo y también de aquella "unanimidad ontosemilógica"2 de las que se pudiera todavía reducir una aspiración universal a la verdad. La contingencia y la ambigüedad se han convertido en la experiencia predominante de la post-modernidad.

A pesar de estos hechos y supuestamente debido al alto grado de necesidad de sentido de los seres humanos en las sociedades modemas, los derechos humanos se han ido convirtiendo en un mito". Los mitos "permiten, en tiempos de crisis, dar un sentido a la actuación del individuo"4. Ellos hacen transparentes las contradicciones sociales y los intereses heterogéneos, proporcionan orientación 
de una manera imperceptible, y les otorgan a los seres humanos un punto sólido de referencia, a partir del cual ellos pueden participar en los procesos de transformación de la sociedad, introduciéndose como actores individuales en ámbitos colectivos. Los derechos humanos indican un objetivo, contienen la visión de una vida humana digna en el medio de toda la creación, liberando así el potencial utópico para poderse comprometer con ese objetivo.

\section{Las tres generaciones de los derechos humanos.}

Para hacernos conscientes de la amplitud, de las posibilidades, y también de los límites de los derechos humanos como aspiraciones universales de validez, es útil esbozar y caracterizar su núcleo y su consiguiente desarrollo en las así llamadas tres generaciones de los derechos humanos.

Sin duda, el núcleo de los derechos humanos consiste en el "derecho al derecho", tal como se menciona en el primer artículo de la Carta de las Naciones Unidas: "todos los seres humanos son libres e iguales en dignidad y derechos".

Como ha mostrado Hermann Pius Siller en la apertura del Simposio de teología intercultural a propósito del tema "Derechos humanos universales en el conflicto de las culturas", "la capacidad para el derecho es independiente de las culturas por que ella no es otorgada por nadie"s. Los derechos humanos individuales, en el trasfondo de experiencias indescriptibles de injustician y de sufrimientos incontables, formulados ante todo como derechos defensivos respecto a las agresiones estatales, constituyen los derechos humanos en la primera generación. De este modo, el derecho al derecho es también "la expresión del ethos actual universal"7. Mientras que los derechos humanos de la primera generación fueron desarrollados porque a los seres humanos se les negó su propia constitución como sujetos de derechos ${ }^{\natural}$, los derechos humanos de la segunda generación surgieron en el trasfondo de las crisis sociales en la Europa de los siglos XIX y $\mathrm{XX}$, y representan la elaboración de estas experiencias de crisis de la modernidad y están formulados como derecho social subjetivo de los individuos frente al estado a partir de la igualdad de todos los seres hümanos. Estos derechos se muestran, por tanto, como soluciones culturalmente condicionadas de problemas a partir de las experiencias concretas de sufrimiento y de crisis de un pueblo". De aquí se sigue que en el caso de los derechos humanos individuales hay que dar cuenta del hecho de sus condicionamientos históricos, siendo necesaria una adaptación a contextos culturales distintos "'. Aquí juegan un rol decisivo las tradiciones e instituciones sociales y las estructuras económicas fácticamente dadas.

Esto puede decirse ante todo del derecho humano al desarrollo, que fue proclamado en la cuadragésimo primera $\left(41^{\mathrm{D}}\right)$ sesión de la nonagésimo séptima $\left(97^{\circ}\right)$ asamblea general de las naciones unidas, el cuatro de diciembre de 1986. "Este derecho al desarrollo" - argumenta Hermann Pius Siller- "es sin duda 
una novedad importante en el desarrollo de los derechos humanos. Ahora bien, en la declaración se habla de desarrollo en singular y de este modo se presupone como obvio que las condiciones antropológicas de partida son las mismas y que por ellos los desarrollos transcurren todas partes con el mismo sentido. Pero precisamente esto es lo que no se puede presuponer... El problema consiste precisamente en la suposición de una igualdad universal de sentido de todos los desarrollos, al estilo del proceso de modernización occidental"'l. Por eso sería bueno hablar de un derecho al desarrollo propio de cada cultura.

\section{El derecho al desarrollo propio de cada cultura}

Esto nos plantea la cuestión en qué medida podemos determinar el derecho al desarrollo propio de cada cultura. A mi modo de ver, el área de teología intercultural en el departamento de teología católica de la Universidad de Frankfurt ha proporcionado, desde sus mismos inicios en el año 1985, algunos importantes resultados investigativos sobre este punto, expuestos en tres simposios internacionales e interdisciplinarios.

El camino que hemos seguido en el diálogo con representantes de otras culturas en torno a los derechos humanos y en especial en la cuestión del derecho humano al desarrollo propio de cada cultura nos condujo a poner el punto de partida en las dificultades de la comunicación intercultural. En este hemos aprendido que el primer presupuesto para la comunicación intercultural es el oír ${ }^{12}$.

Sin oír al otro, no podemos percibir cuales son sus preguntas a nosotros. Comenzamos a hablar muy pronto, tratando de convencer al otro. El otro, al que nos dirigimos, con frecuencia no puede hacer nada con nuestros consejos. Pues se trata de respuestas a preguntas que él nunca se ha planteado. En lugar de caer en la cuenta de que nuestros discursos no le conciernen a nuestro interlocutor, presuponemos que no nos ha oído bien, entonces hablamos más alto, coaccionamos al otro, nos hacemos más intensos en nuestro trabajo para convencerlo, le imponemos formalmente nuestras recomendaciones y finalmente aplicamos la violencia, si es que nuestros consejos, a los que consideramos universalmente válidos, no le atañen. No percibimos, que en realidad nosotros mismos somos los causantes de una incomprensión aparentemente insuperable, porque nosotros no hemos respetado al otro en su alteridad. El resultado de nuestro modo de proceder son necesariamente incomprensiones tanto de la realidad del otro como de la propia realidad. $Y$ es que nos hacemos incapaces de percibir las preguntas que nosotros mismos somos para otros. Lo hacemos, como si nosotros mismos siempre lo hubiéramos entendido todo, y pudiéramos presentar un análisis y dar recomendaciones sin haber escuchado al otro. Pero solamente "donde se interrumpe el propio discurso comienza el oír. La expectativa que olvida todo cálculo, oye, retraída en la palabra, a la palabra que le es dirigida"1.3. Solamente donde llega a mí la oposición del otro a mi pretensión, comienza una comunicación 
que toma en serio al otro como otro. "Al otro como otro solamente estoy abierto cuando en la tranquilidad del oír me deja interpelar por él"14, dice Michael Theunissen en su interpretación de la orientación a la interpelación de Griesbach. Fundamentalmente, la comunicación intercultural presupone que yo cambio mi perspectiva, que cambio mi mirada del otro como una experiencia muy derivada y referida a mí, por una experiencia que parte de mi ser mirado por el otro y que está abierta a mi ser para el otro. Entonces habremos aprendido que nosotros, al escuchar percibimos las molestias que nosotros mismos representamos para otros desde el principio de la modernidad. Nosotros los europeos tenemos la necesidad de dejarnos decir que grandes destrucciones hemos ocasionado en el saber tradicional de otras culturas, y qué grandes anomías hemos causado, solamente mediante las grandes exportaciones de bienes de consumo masivo, en los sistemas eficaces de reglas de otras culturas ${ }^{15}$. Debido a la percepción de las dificultades que nosotros representamos para otros, hemos aprendido a referirnos críticamente a los presupuesto culturales y económicos de nuestro propio desarrollo, a considerar el proceso occidental de desarrollo y modernización desde la perspectiva de otros, y consiguientemente, y a cuestionar en que medida la racionalidad accidental ha liberado potenciales destructivos y en qué medida las consecuencias de estos potenciales destructivos disuelven los fundamentos de nuestra vida social común, poniendo en peligro los fundamentos de nuestra vida natural, y causando también entre nosotros una anomía para cuyo enfrentamiento no hemos elaborado todavía ningún saber social y para cuyo dominio no hemos internalizado todavía individual y culturalmente ningún mecanismo moral de regulación.

Cuando vemos todo esto nos hacemos capaces pára la comunicación intercultural. Entonces aprendemos que la una comunicación intercultural que merezca este nombre comienza a tener lugar cuando nosotros miśmos empezamos a deconstruir esas pretensiones de validez nuestras que siempre intentamos introducir en todos los conflictos interculturales de intereses.

\section{Deconstrucción de las pretensiones de validez universal}

Si en el contexto de las discusiones sobre los derechos humanos partimos de que las pretensiones de validez universal plasmadas en la Carta de las Naciones Unidas, incluyendo el derecho al desarrollo, se deben en realidad al contexto cultural de Occidente, entonces los occidentales debemos escuchar las objeciones que parten de los saberes tradicionales de otras culturas y debemos trabajar, en comunicación cón representantes signficativos de otras culturas, en la deconstrucción de esas pretensiones universales de validez ancladas en nuestra cultura. Todos los interlocutores que han venido a nosotros (a pesar de incontables experiencias dolorosas en el trato con Occidente en diversos niveles) han hecho un esfuerzo enorme para implicarnos en el diálogo sobre las consecuencias catastróficas que la validez de nuestras pretensiones tiene en las estructuras econó- 
micas, culturales y eclesiales de otras culturas. Nos han mostrado con toda evidencia que el potencial destructivo que ${ }^{16}$ liberan los procesos de racionalización de industrialización y de modernización bajo cuya violencia destructiva de las culturas e irrespetuosa de las personas ellos mismos han sufrido ${ }^{17}$, en realidad tampoco se detiene ante nuestras propias fronteras, tal como muestra la codicia consumista y egoísta del dios mercado ${ }^{18}$. Una codicia por la que estamos todos determinados y que determina nuestra relación con nosotros mismos, con nuestros semejantes, con el medio y con la naturaleza. En los diálogos y encuentros interculturales, se me ha hecho claro que nosotros en Occidente tenemos que tomarnos en serio la deconstrucción de nuestras pretensiones de validez universal. Por eso mismo quisiera emprender el intento y reflexionar sobre la deconstrucción de las pretensiones universales de validez, las cuales están entrañadas en nuestro sistema económico y en nuestras estructuras monetarias.

\section{Deconstrucción de las pretensiones universales de validez del Occidente}

El primer ámbito es nuestro sistema económico. En el marco de nuestra pregunta sobre el desarrollo propio de cada cultura fuimos a cada paso confrontados con las pretensiones universales de validez de la economía de mercado, bajo las cuales sufren cuatro quintas partes de la humanidad y bajo las cuales es destruido el futuro mismo de nuestra naturaleza ${ }^{19}$, sometiendo al mundo entero a un inmenso sufrimiento.

Mientras que el socialismo realmente existente no había todavía colapsado, había todavía de hecho en la escena mundial un competidor que concurría con la economía capitalista de mercado. El capitalismo miraba a este competidor, respectivo al cual eran medidos sus resultados. Esta competencia obligaba al capitalismo a mantenerse dentro de unos márgenes, los cuales estaban destinados a proporcionarle una imagen humana y ecológica. En el trasfondo de esta situación global de competencia de pretensiones universales de validez, las clases políticas y los intereses económicos podían comunicarse bajo las pretensiones de validez respectivas, sin que les pudiera ser arrebatada la legitimación política en sus países respectivos. Los países del así llamado Tercer Mundo fueron aplastados mediante la coincidencia de las clases políticas y de los intereses económicos con las pretensiones de validez del sistema capitalista. El tejido social y las tradiciones de esos países fueron sacudidas o destruidas, y ellos fueron declarados, en virtud de las pretensiones de validez de los estados industrializados y económicamente expan'sivos, como "países subdesarrollados".

El mayor impedimento para un mejoramiento significativo de la situación de los derechos humanos en el mundo son el marco de condiciones monetarias y las estructuras que ha determinado el sistema económico neoliberal. Ellas no han caído del cielo y no son inmutables. Son el resultado de procesos sociales en las sociedades de las ricas naciones industrializadas, y no solo pueden, sino que 
tienen necesariamente que ser cambiadas cuando los seres humanos perciben su permanencia como destructiva, y cuando los fundamentos naturales, sociales y culturales de la vida son amenazados. Se extiende el conocimiento de que la forma actual del crecimiento económico ya no promueve el bienestar de los seres humanos y el mantenimiento del medio natural, sino que se convierte en un fin en sí mismo, el cual solamente sirve para el crecimiento de la propiedad monetaria, mientras que de hecho más mengua que aumenta el bienestar bien entendido ${ }^{211}$.

La presión cultural puede obligar a que se realicen las necesarias adaptaciones; una presión que surge de la base, de los desfavorecidos. Ellos constituyen, por así decirlo, la fuerza innovativa y creativa que obliga a que tengan lugar los cambios. La creatividad siempre tiene que ser atacada y transformada por las clases dominantes de la sociedad con el fin de mantener el sistema. Si esto no sucede, el final del sistema está ya programado. Tenemos entonces que preguntarnos: ¿cómo se pueden cambiar las estructuras monetarias en su calidad de zona de fractura del sistema? En principio yo veo cuatro vías:

\subsection{Primera vía: el impuesto Tobin}

La primera vía se mantendría plenamente dentro del marco de las estructuras dominantes. Se trata del impuesto Tobin. Fue sugerido por el economista James Tobin "para que los gobiernos pudieran recuperar su autonomía en las decisiones económicas nacionales" ${ }^{\prime 1}$. James Tobin, quien por cierto recibió el premio Nobel de economía en 1981, recomendó cargar con impuestos el comercio mundial de divisas. Unas cifras aclaran el contexto: "según datos del Fondo Monetario Internacional, el comercio de divisas en 1995 alcanzó una cuantía de 1300 millardos (un billón trescientos mil millones) de dólares, frente a 18 millardos (dieciocho mil millones) de dólares a principios de los años setenta. En comparación, los bienes y servicios que circulan en todo el mundo alcanzan un valor de 4300 millardos ( 4 billones trescientos mil millones) de dólares. Además, los negocios de divisas tienen lugar en un plazo de a lo sumo siete días, y muchos de ellos incluso en el mismo día. En Londres, el más importante centro del comercio de divisas, cuatro quintas partes de las transacciones ya no están en conexión directa con movimientos comerciales o inversiones" ${ }^{22}$. Se trata de especulaciones, las cuales no se traducen positivamente en inversiones en la actividad económica, sino que por el contrario producen ponen en peligro el mercado y la autonomía de la economía del país y de países enteros, sirviendo en último término nada más que al enriquecimiento de los especuladores, sin que a éstos se les exija ninguna obligación social.

El impuesto sobre las divisas tendría un efecto doble. Por una parte se haría justicia a las obligaciones sociales que derivan de la posesión de dinero y de sus consiguientes beneficios. Por otra parte el impuesto sobre las divisas facilitaría 
la afluencia de dinero hacia la actividad económica, íaciendo más financiable la implementación de innovaciones ecológicas y sociales. Finalmente, los negocios a corto plazo se reducirían, y se desarrollarían los negocios a largo plazo, ya que los de corto plazo serían los más afectados por el impuesto Tobin. Ciertamente los réditos serían cuantiosos. "Con una tasa de impuesto del 0.25 por ciento se ingresarían anualmente 290 millardos (290 mil millones) de dólares"-3. Sin embargo, el impuesto Tobin no ha sido implementado, a pesar de que es bien conocido por los ministros de finanzas y los jefes de gobierno de los estados del grupo de los siente, y a pesar de que un grupo de expertos ha puesto de relieve los efectos extraordinariamente positivos que tendría para la economía mun$\mathrm{dial}^{24}$. Esto es especialmente sorprendente teniendo en cuenta que los ingresos conseguidos con el impuesto Tobin podrían reducir la deuda estatal. En lugar de esto, Weigel, Rexrodt y Kohl, al igual que otros estados, permiten que los privilegios del capital especulativo libre de impuestos aumenten las deudas del estado, hundiendo de este modo las prestaciones sociales del mismo.

\subsection{La segunda vía: la transformación del sistema de intereses}

La segunda vía sería el cambio de las estructuras monetarias. Esta vía implica una transformación fundamental de las condiciones mismas del orden monetario, es decir un cambio del sistema de intereses. Con otras palabras: "la formación de reservas (especulativas) de liquidez debe ser dificultada cargando con gastos extraordinarios de por ejemplo el $4 \%$ de la contabilidad media a todos aquellos que no reinvierten sus ingresos en consumo, inversiones o en cuentas de ahorro. Este modo de asegurar la circulación impidiendo la formación de reservas en el círculo de la actividad económica ya no tiene como molor el premio producido por los intereses, sino el castigo eficaz de todo retiro de liquidez monetaria fuera del círculo de la actividad económica" ${ }^{29}$. Pensando en categorías éticas, esto se justifica por el hecho de que el dinero públicamente reconocido como dinero (es decir, garantizado socialmente, como ya se ha explicado) representa una institución social. En esa medida, el dinero puede asumir las funciones de un medio cambio y de un medio de acumulación de valor, adquiriendo, por el hecho de poder ser cambiado en cualquier momento por un producto, un valor universal para cualquiera que está en posesión del mismo. Y es que, para el poseedor de dinero, ni se presentan gastos de almacenamiento, ni el dinero mismo se estropea cuando es acumulado, a diferencia de lo que les sucede a las papas del campesino, el cual esta obligado a transformarlas en dinero lo más rápidamente posible si no quiere arriesgarse a experimentar pérdidas debido a que las papas se envejecen o se estropean. Esto tiene por consecuencia el que el dinero sea muy deseado, de modo que puede ser prestado temporalmente como crédito a cambio de intereses o de intereses sobres intereses a aquellos que no lo tienen. Esto es muy distinto a lo que sucede al campesino. Cuando él presta un saco de papas, está contento cuando tras un tiempo arbitrariamente 
estipulado recibe de vuelta exactamente el mismo saco, y no más. Pierre Joseph Proudhon (1809-1865), un contemporáneo de Karl Marx (1818-1883) cayó en la cuenta de este problema y a diferencia de Marx consideró la plusvalía como consecuencia de la circulación monetaria. "Negocios cambiarios y crediticios en los que aparece el interés, son manifestaciones de la esfera de la circulación"h, dice Dieter Suhr siguiendo a Proudhon. Y esto significa que el verdadero capitalista no es el empresario ni el "capitalista industrial", sino el capitalista financiero, quien ingresa intereses e intereses de intereses mediante la ventaja de comodín que tiene el dinero en cuento medio universal de cambio y de comunicación, no prestando absolutamente ninguna contribución al incremento del valor.

Para un cambio de las estructuras monetarias falta actualmente todavía la necesaria conciencia del conjunto de la sociedad. Los responsables en las iglesias deberían comprometerse en que todas las fuerzas fueran movilizadas para un cambio en las estructuras monetarias. Aunque en la actualidad no está suficientemente difundida la conciencia para lo que yo he llamado la vía radical, podría formarse rápidamente debido a la creciente presión social, ecológica y cultural. Por otro lado, muchos piensan que las imbricaciones mundiales de nuestro sistema constituyen un impedimento que condena al fracaso un camino aislado de la República Federal Alemana, por más que existan algunos intentos exitosos limitados al ámbito local.

\subsection{Tercera vía: la movilización ética de los sujetos económicos.}

Es lo que llamo la vía suave o moderada. Esta vía cuenta con la posibilidad de movilizar a los implicados, y apuesta por ellos. Ciertamente, esto exige un cambio en la conciencia y una posibilidad de movilizar tanto a los consumidores como a los empresarios y a los inversores, a los propietarios de capital financiero y a los bancos. Todos ellos no pueden seguir permitiendo que el dios en torno al que nuestra sociedad se mueva sea el dinero. No nos está permitido dejarnos dictar por el dios Mammon cuáles sean los problemas que tenemos que resolver. Tenemos que combatirle al dios Mammon la definición de los problemas. Con otras palabras: no podemos seguir permitiendo que los empresarios y los banqueros vengan y digan: "primero deben estar claras las cuentas, primero hay que lograr beneficios y entonces podremos hablar de moral". Esta receta, afirmada absolutamente, es económicamente miope y conduce a un camino sin salido. No puede ser serio que la tarea del $90 \%$ de la población consista en entregarse como víctimas para que Jas necesidades de crecimiento exponencial del dinero puedan ser satisfechas. Los seres humanos no existen para el dinero, sino que el dinero existe para servir a los seres humanos en su preocupación y esfuerzo por una existencia digna de toda la humanidad. De ahí se sigue que las víctimas de nuestro marco monetario actual, los desempleados, los empresarios endeudados, los pobres en el mundo entero y el dañado medio ambiente tienen que recibir la primacía en la definición del problema. La primera pregunta no es "¿Qué es 
bueno para el gran dinero?, sino ¿Qué es bueno para los seres humanos y para toda la creación?.

Pero, ¿cómo puede funcionar esto? La primera condición es un interés ampliamente difundido de las personas en el mantenimiento de la economía social de mercado. Yo pienso que este interés esta ahí. Pero, ¿no hay otra alternativa que fuera mejor? En nuestro orden constitucional democrático y en el consiguiente saber social de nuestra cultura hemos plasmado una forma de relaciones y condiciones sociales en la que debería ser alcanzado un equilibrio entre la actividad económica y el estado, al servicio de una vida social común humanamente digna y al servicio del mantenimiento de los fundamentos naturales de la vida. A partir de esta orientación fundamental tendrían que ser ordenadas las condiciones reales del estado y de la economía, y en vistas a esta orientación fundamental tendrían ellas que justificarse. Con otras palabras: el estado no puede ser puesto sin más al servicio de las estructuras económicas y monetarias. Al estado siempre se le ha de preguntar en que medida, frente a la presión de los propietarios de dinero y frente a la presión del capital, cumple su tarea más propia de poner las condiciones para que una vida social común humanamente digna sea posible, asegurando los fundamentos naturales y culturales de la vida. La creación de zonas libres de comercio y la ampliación de los mercados hasta su globalización, tal como sucede en la OMC, deben proporcionar la prueba de que no sirven a los intereses del dinero, sino a los de las personas, pudiendo garantizar la realización humana en comunidad ${ }^{27}$. A mi modo de ver, esto es perfectamente realizable si se utilizan las posibilidades democráticas y se crean estructuras de supervisión que controlen la política de negocios de los bancos para que las relaciones de producción sean configuradas mediante flujos de dinero de tal modo que se posibilite un desarrollo permanente de la sociedad mundial en el que la sostenibilidad natural, social y cultural funcionen como criterios de primer orden. Yo estoy de acuerdo con la tesis de Joachin Hirsch según la cual "el capitalismo puede ser transformado de un modo permanente si se consigue que los procesos de producción, de inversión y de mercado sean sometidos a un control efectivo público y democrático. Es pensable un modelo de sociedad que permita la revalorización del capital y los beneficios, pero de una manera políticamente y socialmente regulada. No se trata tanto de una cuestión sobre las relaciones formales de producción, sino más bien de estructuras sociales y políticas de poder"2r.

Existen propietarios de grandes sumas de capital monetario que han captado que una acumulación continuada de dinero solamente conduce a un callejón sin salida. En consecuencia reducen sus montañas de dinero entregando cantidades considerables en beneficio de la comunidad nacional o internacional mediante fundaciones u otras formas de usufructo. Crece el número de empresarios motivados éticamente que introducen productos y formas de producción sostenibles natural, social y culturalmente. El desarrollo técnico, los sistemas económicos y 
las estructuras monetarias no caen del cielo. No son realidades perfectas ni inmutables, sino el resultado de procesos sociales en una cultura. La cultura es transformada por ellos, pero al mismo tiempo ella posee en su saber social la fuerza para transformar la sociedad y la técnica cuando su permanencia es percibida por los seres humanos como destructiva, y cuando los fundamentos naturales y sociales de la vida son puestos en peligro.

Se extiende el conocimiento de que la forma actual de crecimiento económico ha dejado de servir al bienestar de los seres humanos y de su medio natural. El crecimiento económico se ha convertido en un fin en sí mismo que solamente sirve a la acumulación de dinero, mientras que el bienestar bien entendido es más impedido que apoyado (Scherhorn y otros, 1997). Esta afirmación es válida en el plano nacional, en el plano europeo y en el plano global. De este modo crece la conciencia de que todas las fuerzas sociales necesitan ser movilizadas, para producir una presión cultural tal que capacite a la economía social de mercado para mediar entre el liberalismo y el socialismo, entre la competencia y la solidaridad y entre la economía y la ecología.

Sin duda no se puede alcanzar un cambio fundamental de nuestro sistema económico de la noche a la mañana. Pero hay flujos monetarios que podrían ser canalizados de forma tal que impulsen pequeños cambios en dirección hacia innovaciones social, natural y culturalmente sostenibles. De este modo contribuirían a poner bajo control los potenciales destructivos de nuestro sistema económico.

Las agencias dedicadas a clasificar las empresas en relación a su responsabilidad financiera y su confiabilidad (rating), tales como Standard \& Poor's, Moody o EuroRating, utilizan ante todo criterios financieros como la "bonanza", la "rentabilidad", los "costes de transacción", "el tiempo de circulación", y los "aspectos impositivos". Pero, con el trasfondo de la presión cultural, va creciendo el interés en las inversiones monetarias que contemplan aspectos éticos. Una creciente minoría de inversores parte de la premisa de que la propiedad obliga (Roche, Hoffmann \& Homolka 1992).

Esta tendencia tiene un impulso adicional en la medida en que crece la convicción de que a largo plazo solamente empresas éticamente "limpias" lograrán imponerse de una manera convincente en el mercado, de modo que ellas proporcionarán mayores beneficios que otras inversiones, aunque solamente sea por el hecho de que estas empresas están anticipando futuras regulaciones legales. Los inversionistas son guiados en esto por investigaciones que han podido mostrar que una inversión ética tiene mayores rendimientos promedio que otra inversión normal ${ }^{24}$. En el ámbito de la crisis bursátil de 1987 esto ya se verificó en el mercado norteamericano, puesto que en ningún fondo ético de inversiones tuvieron lugar pérdidas, a diferencia de lo que usualmente sucedía. 
La decisión a favor de criterios éticos puede tener enormes consecuencias. Con ayuda de la clasificación ética de los negocios pueden impulsarse en pequeñas y medianas empresas cierto tipo de innovaciones técnicas que no tendrían ningún sentido desde el punto de vista de criterios puramente económicos a corto plazo. Esto tiene un efecto considerable para un desarrollo empresarial ecológicamente orientado y para una protección preventiva del medio ambiente. Son precisamente las pequeñas y las medianas empresas las que tienen un potencial creativo y una flexibilidad que les permite, si hay capital dispuesto a ser invertido, la iniciación de nuevos caminos en el desarrollo sostenible de productos, en las técnicas productivas y en la prestación de servicios. Uno puede esperar que en el futuro también en Alemania estarán mas representadas en la bolsa.

Considerado desde un punto de vista filosófico, las inversiones éticas constituyen acciones que no son estrictamente obligatorias, pero que tampoco son supererogatorias. Se podría tratar de deberes imperfectos en el sentido de Kant, los cuales por definición dejan cierto margen para determinar cómo se podrían realizar. Los modos de actuar que unen inmediatamente las inversiones éticas a las inversiones de capital constituyen un indicio de que las inversiones éticas son percibidas por muchos como un deber moral imperfecto. No sería necesario tener mala conciencia sino todo el capital propio se invierte de un modo ético.

El problema consiste, por tanto, en elaborar el proyecto de una fundamentación teórica adecuada que no introduzca premisas éticas o económicas que sean discutibles incluso para los representantes de la clasificación ética y ecológica de las empresas. Un hilo conductor para la valoración ética de las empresas plantea importantes preguntas de filosofía moral y también preguntas criteriológicas, pero no puede utilizar un lastre teórico inútil ${ }^{311}$.

\subsection{La cuarta vía: evitar el lavado de dinero.}

La cuarta posibilidad de prestar una contribución esencial a la realización de los derechos humanos es impedir el lavado de dinero, en el que está envuelto nuestro sistema económico, los bancos, etc., y del que ellos extraen beneficios, aunque también puede volverse peligroso para ellos mismos. Puede que a algún lector esta tesis le parezca traída por los pelos. Pero algunas indicaciones pudieran hacerla comprensible. El lavado de dinero es definido en el informe de investigación de la President's Commissión on Organized Crime del año 1984 como un proceso que esconde fuentes o utilizaciones ilegales de ingresos para después presentar estos ingresos como obtenidos legalmente ${ }^{31}$. Según Thomas Achim Werner, el lavado de dinero es "la reconducción hacia el circuito financiero legal de propiedades obtenidas ilegalmente. El objetivo es el ocultamiento de su origen y la liberación de esos valores respecto a cualquier posibilidad de intervención por parte de las autoridades criminales. Al mismo tiempo se pretende evitar la prosecución criminal de aquellas personas que han realizado los 
hechos criminales"32. De este modo hemos mencionado algunos términos clave, sobre los cuales hay que hacer un par de observaciones.

Hay en primer lugar hechos criminales mediante los cuales se hacen ganancias ilegales. Entre ellos destaca ante todo el tráfico ilegal de drogas, que rinde anualmente en el mundo entre 500 y 800 millardos de dólares $(500$ a $800 \mathrm{mil}$ millones de dólares) "El tráfico de drogas representa el mayor negocio mundial neto":3. Entre los hechos criminales se cuenta también el tráfico de seres humanos, mediante el cual "mujeres de los países pobres mediante promesas falsas son introducidas de contrabando en los países industrializados y obligadas a prostituirse":34. También hay que contar "el contrabando de niños para adopción, al igual que el tráfico ilegal de órganos, el cual esta parcialmente ligado a la sustracción de órganos a personas secuestradas o asesinadas" ${ }^{35}$. Además hay que tener en cuenta el tráfico de armas, la criminalidad ecológica, los pagos a cambio de "protección", etc.

Prescindiendo de los hechos criminales mencionados, es necesario también considerar aquí las prácticas inhumanas y explotadoras de los traficantes de drogas respecto a los campesinos que cultivan la coca, tal como por ejemplo sucede en Bolivia y en otros países del Tercer Mundo.

¿Dónde están las causas? Cuando los Estados Unidos y otras naciones industrializadas occidentales rechazan toda culta y exigen el fin de la producción de coca, estamos ante algo que es hipócrita en tres sentidos. En primer lugar, los consumidores de droga están sobre todo en los países ricos. En todo el mundo hay unos 12 millones de consumidores de cocaína y 8 millones de consumidores de heroína. Para mencionar un ejemplo: "en relación al número de habitantes, Suiza arroja en el contexto europeo la mayor cuota de muertos por drogas, y de condenados y sospechosos según la ley de estupefacientes":3h. En segundo lugar, la causa de la creciente producción de drogas es la explotación de los países del Tercer Mundo por parte de los países industrializados, y también el endeudamiento y sobre-endeudamiento de los países pobres inducido por los países ricos. En tercer lugar y por último, una de las causas más importantes para el floreciente tráfico ilegal de drogas es el hecho de que con la ayuda de instituciones financieras de renombre funciona un lavado de dinero que permite introducir sin muchas dificultades el dinero ganado ilegalmente en el círculo monetario legal. Thomas Achim Werner nombre los actores del lavado de dinero:

1) Los miembros del crimen organizado que realizaron los hechos criminales y obtuvieron ganancias se comportan de un modo conspirativo y llevan a cabo ellos mismo el lavado de dinero. Aquí hay que contar el contrabando físico de dinero a través de las fronteras, en parte con los mismos medios y métodos con los que se lleva a cabo el contrabando de drogas.

2) Personas que consciente o inconscientemente trabajan con los grupos criminales, pero que por lo demás se mueven en un medio legal y por tanto 
resultan confiables y nada sospechosos para los bancos y otras instituciones. Un ejemplo son los mensajeros, los corredores de divisas, los empleados corruptos del banco, los notarios y los abogados de confianza del crimen organizado. También hay que contar los expertos en finanzas y los lavadores especializados de dinero, los cuales prestan servicios muy bien pagados al crimen organizado.

3) Personas que en el ejercicio de sus ocupaciones legales son utilizadas para el lavado de dinero, con frecuencia sin ellos saberlo. Se trata de empleados de banco, de mensajeros comerciales, de abogados, de gerentes, de numerosos financieros, de sociedades de inversión, de comerciantes de inmuebles, etc.

El comportamiento criminal se localiza en los dos primeros grupos, mientras que el tercero suele estar implicado de un modo inconsciente o a lo sumo negligente ${ }^{37}$.

Resumiendo puede decirse que para el éxito del lavado de dinero es imprescindible la participación del sistema bancario. "Debido a su posición central en el sistema financiero y crediticio, en la administración de la propiedad y en el comercio (internacional), los bancos funcionan como puestos centrales del lavado de dinero. Ninguna otra institución puede transferir y transformar tan rápidamente toda la masa de dinero que se desea legalizar"38. Un rol especial en el lavado de dinero lo desempeñan los oasis de impuestos y los bancos offshore. Aquí se dan condiciones óptimas para el lavado de dinero, tales como la casi inexistencia de impuestos, ninguna obligación de llevar una contabilidad, una supervisión de los bancos casi nula, un rechazo de la ayuda jurídica internacional, un secreto bancario estricto, un comercio libre de divisas, etc. Junto a Luxemburgo, Lichtenstein, Monte Carlo, Suiza, Austria, y otros, "son las Islas Caimán, un grupo de islas en el Caribe con 14000 habitantes, uno de los lugares offshore más importantes. Solamente aquí están registrados varios miles de compañías offshore y unos 500 bancos" "39, entre los que se encuentran todos los grandes bancos alemanes incluyendo el Banco Federal Alemán. El Fondo Monetario Internacional consideraba los fondos bancarios de los siete lugares financieros offshores más importantes en 973.1 millardos (973 mil 100 millones) de dólares, lo cual significa "más de una quinta parte del monto total de fondos bancarios" 4 ". En Alemania se lavan según estimaciones actuales entre 50 y 89 millardos (50 a 89 mil millones) de marcos alemanes. En este punto es admirable que muchos bancos, buscando ganancias por intereses a corto plazo, colaboran en el lavado de dinero, a pesar de que con ello favorecen el ascenso del crimen organizado, que de este modo logra reunir grandes montos de capital en el sector legal, lo cual acaba significando un peligro para los bancos mismos y para economías nacionales completas. $\mathrm{Y}$ es que "estos dineros lavados pueden por una parte ser integrados de un modo seguro, legal y rentable, mientras que por otra parte pueden ser utilizados como capital especulativo para ejercer presión 
económica y política"41. De aquí surgen los peligros para la sociedad, para la democracia y para el orden jurídico, puesto que se reducen las barreras para el soborno, y para la cooperación con el crimen organizado y con el crimen económico. Las naciones industrializadas y los bancos que se encuentran en ellas deberían, solamente en virtud del propio interés, poner todas las dificultades al lavado de dinero, favoreciendo la posibilidad de un descubrimiento del origen de los dineros ilegales. El crimen organizado y el desprecio de los derechos humanos que se une a él en todo el mundo no se puede impedir solamente impidiendo el cultivo de las plantas de las que no se obtienen las drogas. Todo lo contrario: además de impedir el lavado de dinero como punto de partida decisivo para combatir el crimen organizado, son necesarias también una serie de medidas que han de ser financiadas por los países ricos. Las actuaciones de la así llamada "política de desarrollo" de estos países han tendido desde los años setenta ante todo a orientar las economías de los países del Tercer Mundo a los intereses de revalorización de las propias economías. De este modo son los causantes de la destrucción de las economías tradicionales de subsistencia de estos países y del endeudamiento y sobreendeudamiento de los países del así llamado Tercer Mundo.

También Werner subraya esta relación: "para reducir el cultivo de drogas y la consiguiente oferta se necesitan medidas amplias, puesto que el cultivo está inseparablemente conectado con la situación de los países del así llamado Tercer Mundo cuyo endeudamiento representaba al final de los años ochenta la suma de 1300 millardos (un billón 300 mil millones) de dólares. A causa de la caída de los precios del mercado mundial de materias primas y de productos agrícolas, el cultivo de la coca y de la adormidera representa la última posibilidad económica para los campesinos. Mientras que con el cultivo de la coca y de la adormidera consigan beneficios más altos que con otros productos agrícolas, solamente podrán ser disuadidos del cultivo de las drogas con medidas de ayuda eficaces. Posibilidades para tal ayuda son por ejemplo los precios justos para las materias primas y un comercio justo en el que no solamente consigan beneficios las empresas multinacionales, sino también los campesinos. El intento de reducir el cultivo de la droga mediante las presiones de los países industrializados sobre los países de cultivo está con toda probabilidad condenado al fracaso mientras las relaciones económicas, la amenaza del desempleo y los problemas sociales no sean considerados suficientemente. Una solución efectiva exige medidas solidarias que pueden ser útiles también a los países de cultivo"42.

En palabras claras, esto significa que las naciones industrializadas, por razón de su envolvimiento causal, tienen la responsabilidad principal en la desolada situación actual de los países del así llamado Tercer Mundo, en su endeudamiento, en su pobreza, en su hambre y en el crecimiento del crimen organizado.

Por tanto esas naciones están obligadas a comprometerse con todos los medios a su disposición en las siguientes medidas: 
1) Impedir el lavado de dinero creando condiciones monetarias efectivas y leyes que se puedan implementar.

2) Condonar completa e inmediatamente las deudas de todos los países del así llamado Tercer Mundo.

3) Ayudar a crear una economía de subsistencia sostenible en estos países.

\section{Notas}

1. Cf. Zygmunt Bauman, Moderne un Ambivalenz. Das Ende der Eindeutigkeit, Hamburg, 1992.

2. Jochen Hörisch, Kopf oder Zahl. Die Poesie des Geldes, Frankfurt, 1996, p.26

3. En el libro de Baumann sobre Postmoderne Ethik (Hamburg, 1995, p.13) se puede leer además:

"Pienso que lo nuevo del punto de partida postmoderno en ética no consiste primeramente y ante todo en el abandono de temas morales característicos de la modernidad, sino en el rechazo de modos típicamente modernos de enfrentar los temas morales (a saber, el hecho de reaccionar a los desafíos de la praxis política con regulaciones normativas coactivas, buscando filosófica y teóricamente el absoluto, los universales o fundamentaciones teóricas últimas). Los grandes intereses de la ética (como los derechos humanos, la justicia social, el balance entre la cooperación pacífica y la afirmación individual, la sincronización del comportamiento individual y el bien colectivo) no han perdido nada de su actualidad. Pero ellos tienen que ser vistos y enfocados de otra manera.

4. Gotthard Fuchs y Bernhard Moltmann, "Mythen der Stad", en Gotthard Fuchs, Bernhard Moltmann y Walter Prigge (eds), Mythos Metropole, Frankfurt, 1995, pp.919, aqui p.18.

5. Hermmann Pius Siller, "Die Beiträge der vielen besonderen Lebensformen zu dem einen Mens chenrecth", en Johannes Hoffann (ed.), Universale Menschenrechte im Widerspuch der Kulturen, vol.2: Das eine Menschenrecht for alle und die vielen Lebensformen, Frankfurt, 1994, pp.13-24, aqui p.14.

6. Cf. Judith N. Shklar, Über Ungerechtigkeit. Erkundungen zu einem moralischen Gefiihl, Berlin, 1992, p.135.

7. Johannes Schwartländer, "Menschenrechte -eine Herausforderung der Kirche", en id. (ed.), Menschenrechte -eine Herausforderung der Kirche, Mainz, 1979, pp.15-57, aquí p.15.

8. Cf. Martin Honecker, “Universalität und Unteilbarkeit der Menschenrechte?", en Lothar Brock (ed.), Menschenrechte und Entwicklung. Beiträge zum ökumenischen und internationalen Dialog, Hannover-Frankfurt, 1996, pp.20-30, aqui p.28 Honecker afirma: "los derechos humanos presuponen un derecho fundante, el derecho de todo ser humano a tener derechos (Hannah Arendt). A pesar de todas las barreras con las que choca la realización de los derechos humanos individuales, hay un derecho inalienable, que es que todo ser humano debe ser, como ser humano, sujeto de derecho".

9. Cf. L. Brock, Menschenrechte im Nord-Süd-Vernaltnis, en id. (ed.), op. cit., pp 1119, aquí p. 16:

"La comprensión occidental de los derechos humanos fundamenta en primera línea derechos defensivos y derechos subjetivos del ciudadano respecto al estado. Sin em- 
bargo, la política internacional de derechos humanos es en primera línea política estatal. Un grupo de estados requiere el cumplimiento de los derechos humanos por otro estado o un grupo de estados. Este es uno de los motivos de los problemas de credibilidad de la política internacional de derechos humanos.

10. Cf. Johannes Hoffmann, Das cine Menschenrecht für alle und die viclen Lebensformen. Eine Ploblemskizze, en id. (ed.), Begründung von Menschenrechten aus der Sicht unterschiedlicher Kulturen, vol. 1: Das eine Menschenrecht für alle und die viclen Lebensformen, Frankfurt, 1991, pp. 17-33, aqui pp. 28-29.

11.H.P. Siller, "Die Frage nach kilturcigenen Rationalitäts- und Entwiclungspotentialen", en J. Hoffmann (ed.), Die Vernunft in den Kulturen. Das Menschenrecht auf kultureigene Entwickiung, vol. 3: Das eien Menschenrecht für alle und viclen Lebensformen, Frankfurt, 1995, pp. 39-55, aquí p. 39.

12. El condicionamiento político de la ayuda al desarrollo, si no considera esto, tiene necesariamente que fracasar desde un principio. Cf. Franz Nuscheler, "Menschenrechtsschutz durch Konditionierung der Entwicklungshilfe?”, en L. Brock, Menschenrechte und Entwincfung, op. cit., pp. 65-72, aqui pp. 67-68. Sobre la carencia de competencia para actuar interculturalmente en el trabajo de ayuda al desarollo nacional e internacional, señala Hermann Sauter, director del Instituto Iberoamericano para Investigación Económica de la Universidad de Göttingen: "No se exagera cuando se dice que la existencia y el contenido del pacto social ha sido desconocido durante largo tiempo por las organizaciones donantes en el trabajo de ayuda al desarrollo bilateral y multilateral. Solamente en el contexto de la "cumbre social mundial" del año 1995 esto ha comenzado a cambiar un poco. Sintomático de este desconocimiento es que un trabajador del banco mundial, preguntado sobre el pacto social, hasta muy recientemente bien podria haber dicho que el nunca habia oido hablar de ese pacto", Hermann Sautter, "Soziale Menschenrechte und Entwicklungszusammenarbeit: Von der Konvergenz der Ziele zur Komplementarität der Mittel", en L. Brock, op. cit., pp. 181-193, aquí p. 185.

13. Eduard Grisebach, Gegenwart. Eine Kritische Ethik, Halle, 1928, p.577.

14. Michael Theunissen, Der Andere. Studien Zur Sozialontologie der Gegenwart, Berlin, 1965 , p.365.

15. Según Peter Atteslander "la anomía significa una real o aparente ausencia de salidas, en el contexto de una situación social donde el propio desarrollo esta impedido o imposibilitado", Peter Atteslander, "Kulturelle Eingenentwiclung als Kampf gegen Anomie", en id. (ed.), Kulturelle Eingenentwiclung, Frankfurt, 1993, pp. 13-32, aquí p. 13.

16. Cf. Tzvetan Todorov, Die Eroberung Amerikas. Das Problem des Anderen, Frankfurt, 1985; Enrique Dussel, Von der Erfingung Amerikas zur Etdeckung des Anderen Ein Project der Transmoderne, Düsscldorf, 1993.

17. Jon Sobrino escribe lo siguiente en su libro Sterben ub, wer an Götzen rührt. Das Zeugnis der ermordeten Jesuiten in El Salvador: Fakten und Überlegungen, Frivurg, 1990, p.55: "Yo entiendo que para los ciudadanos del primer mundo es difícil comprender la gravedad de la tragedia. Y es que para aquellos que tienen vida y libertad, es naturalmente difícil captar lo que significan la pobreza y la represión en los países del tercer mundo. Por eso mismo cllos tienen la tendencia a ignorar esta realidad, hacerse los desentendidos y callar. Tal vez ellos callan también porque ellos tienen un sentimiento de culpa inconsciente: uno no puede vivir en la abundancia, tenerlo prác- 
ticamente todo, y querer sicmpre tener más mientras cada día muchos millones de personas mueren de hambre. El conjunto de estos modos de actuar y de estas omisiones es justamente lo que mata a los pobres y a aquellos que los defienden. Por cso mismo la pregunta por los asesinos se dirige a todos nosotros."

18. Cf. Hugo Assmann - Franz Hinkelammert, Götze Markt, Düsseldorf, 1992; F. Hinkelammert, Dite ideologischen Waffen des Todes. Zur Metaphysik des Kapitalismus, Freiburg-Münster, 1985.

19. No faltan investigaciones que muestran al agudización dramática de los problemas sociales y ecológicos. En representación de muchos otros quisiera nombre solamente uno: el Human Development Report 1992, UNDP, New York-Oxford, 1992.

20. Cf. Gerhard Scherhorn y otros, Wohlstandkosten und verantwortliches Handeln. Ergebnisbericht über eien experimentelle Studie im Forschungsschwerpunkt "Mensch und Globale Unmweltveränderungen" der Deutschen Forschungsgemeinschaff, Arbcitspapier 68, Stutgart, 1997, Universät Hohenheim, Lehrstuhl für Kosumtheoric und Verbraucherpolitick.

21. Ibrahim Warde, "Dic Tobin Steuer - ein wening Sand im Getrieve" en Le Monde Diplomatique-Dic Tageszeitung, 12-2-97, p.1.

22. Ibid.

23. Ibid.

24. Cf. Mahbub ul-Haq, Inge Kaus, Isabelle Grunberg (eds.), The Tobin-Tax: Copin with Financila Volatility, Oxford (Oxford University Press), 1996.

25. Ernst Winkler, "Von einer mutation unseres Wirtschaftssystems", en SozialökonomischeArbeits-texte 3, Lütjenburg, 1994 (2. Ed.), p.16.

26. Dieter Suhr, Befreiung der Markwirtschaft vom Kapitalismus. Monetäre Studien zur socialen, ökonomischen und öklogischen Vernunft, Berlin, 1986, p.14.

27. Cf. Enrique Dussel, "Bye bye Weltmarkt? Frehandel oder Regionalisierung des Weltmarktes: Das Frehandelsabkommen zwischen Kanada, México und den USA", en PROKLA, op. cit., 129-156.

28. Joachin Hirsch, Kapitalismus ohne Alternative? Materialisfische Gesellschaftstheoric und Möglichkeiten einer sozialistischen Politik heute, Hamburg, 1990, p.181.

29. Cf. Sandra Schuffelen, "Oko-Aktien überzeugen an der Börse. Natur-Aktien-Indes/ Kursgewinn von round 34 Prozent in zwölf Monaten", in Handelsblatt, 16-4-98, núm. 73, p.41.

30. Johannes Hoffman, Konrad, Ott, Gehard Scherhorn (eds.) Ethische Kriterien für die Bewertung von Unternehmen, Frankfurt-Hohenheimer Leifaden, Dutsch und Englisch, Frankfurt 1992, pp. 11-15.

31. Cf. Mark Picth (ed.) Bekämpfung der Gelwäscherei: Modellfall Scheweiz, Basel 1992, citado en Thomas Achim Werner, Wachstumsbranche Geldwäsche. Die Ökonomisierung der Organisierten Kriminalität, Berlín 1996, p.13.

32. Werner, op. cit., p.14

33. Ibid., p.20

34. Ibid., p. 22

35. Ibid., p.22

36. Ibib., p.130

37. Ibid., p.31

38. Ibid., p.31 
39. Ibid., p.35. Werner cita aquí el anuario de 1998 de la cámara de comercio de las Islas Caimán:

"Los Bancos Deutsche Bank, Dresdner Bank, Commerzbank, BfG, Bayerische Hypothekenund Wechselbank, Bayerische Vereinsbank, Berliner Handelsbank, Frankfurter Bank, Deutsche Bundesbank están representados por la Deutsche Girozentrale Oversca Ltd."

40. IWF International Financial Statistics (1998), citado en Werner, op. cit., p.35.

41. Werner, op. cit., pp. 77-78

42. Ibid., p.105.

Digitalizado por Biblioteca "P. Florentino Idoate, S.J." Universidad Centroamericana José Simeón Cañas 